\title{
Half-metallic behavior of a ferromagnetic metal monolayer in a semiconducting matrix
}

\author{
S. Caprara ${ }^{1,2(a)}$, V. V. Tugushev ${ }^{1,3}$, P. M. Echenique ${ }^{1,4}$ and E. V. Chulkov ${ }^{1,4}$ \\ ${ }^{1}$ Donostia International Physics Center (DIPC) - P. de Manuel Lardizabal 4, 20018, San Sebastián, Basque Country, \\ Spain, EU \\ ${ }^{2}$ CRS SMC, CNR-INFM and Dipartimento di Fisica, Università di Roma "La Sapienza" - Piazzale Aldo Moro, 2, \\ 00185 Roma, Italy, EU \\ ${ }^{3}$ RRC Kurchatov Institute - Kurchatov Sqr. 1, 123182 Moscow, Russia \\ ${ }^{4}$ Departamento de Física de Materiales, Facultad de Ciencias Químicas, UPV/EHU and Centro Mixto \\ CSIC-UPV/EHU - Apdo. 1072, 20080 San Sebastián, Basque Country, Spain, EU
}

received 29 September 2008; accepted in final form 19 December 2008

published online 28 January 2009

PACS 73.40.Sx - Metal-semiconductor-metal structures

PACS 75.70. Cn - Magnetic properties of interfaces (multilayers, superlattices, heterostructures)

PACS 75.10.Lp - Band and itinerant models

\begin{abstract}
We propose a model to describe the formation of a spin-polarized half-metallic state in the proximity of a ferromagnetic metal monolayer embedded into a semiconducting host. The main physical ingredients of our model are the Coulomb interaction between two electrons at the same metallic site, the charge redistribution around the monolayer and the hybridization between the electron states of the metal and of its host. We discuss the phase diagram of the model and the relevance of our results in connection with experimental and numerical results on digital magnetic heterostructures.
\end{abstract}

Copyright (C) EPLA, 2009

Introduction. - Heterostructures in which (sub)monolayers of a transition ferromagnetic metal (FM) are embedded into a semiconductor (SC) host are now the object of an intense investigation in view of possible applications to spintronics. These FM/SC heterostructures can be realized either with single FM (sub)monolayers, or with periodic arrays of coupled FM (sub)monolayers, the so-called digital magnetic heterostrucutres (DMH). Most experimental studies are devoted to systems in which Mn (sub)monolayers are introduced into a III-V $\mathrm{SC}$ (GaAs or GaSb). These studies show that shortor long-range ferromagnetic order exists both in the case of a single (sub)monolayer [1] and in the case of $\mathrm{DMH}[2]$. Little attention has been paid, so far, to FM/SC heterostructures with a SC belonging to the IV group.

On the other hand, $a b$ initio calculations have been applied to DMH, with a periodic array of Mn monolayers along the (001) direction of a III-V SC (Mn/GaAs [3]) and of a IV-group $\mathrm{SC}(\mathrm{Mn} / \mathrm{Ge}[4]$ or $\mathrm{Mn} / \mathrm{Si}[5,6])$. All these calculations put in evidence three important facts: i) ferromagnetism is more favorable than antiferromagnetism in the Mn layers; ii) the charge carriers are confined near

\footnotetext{
(a) E-mail: sergio.caprara@roma1.infn.it
}

the Mn layers; iii) the electron spectrum is half-metallic, with strongly spin-polarized nearly two-dimensional (2D) energy bands. The question then arises about the common physical origin of these facts and their connection to some peculiarity of the geometry and content of the DMH. Indeed, it is well known that half-metallicity is an exception rather than the rule in three-dimensional (3D) FM, where narrow $d$ bands coexist with wide $(s, p)$ bands, for which the full exchange splitting is difficult to realize.

To elaborate on the outcomes of the above-mentioned $a b$ initio calculations and clarify the intimate relation that connects ferromagnetic ordering, the formation of $2 \mathrm{D}$ bands, and half-metallicity, in this letter we use an Anderson-like model. Rather than providing an accurate description of the band structure of a $\mathrm{FM} / \mathrm{SC}$ system, our model is aimed at capturing the essential ingredients, as suggested by numerical calculations, i.e., the strong Coulomb interaction between $d$ electrons, the charge-carrier redistribution and confinement near a FM monolayer, and the hybridization between the $(s, p)$ orbitals of the SC and the $d$-orbitals of the FM. Despite its relative simplicity, our description allows for transparent analytical results, which share many characteristics in common with the issues of numerical calcuations, with 
the advantage that the model parameters can be varied independently, and a phase diagram can be obtained. We show that a generic half-metallic behavior is indeed made possible near a FM layer by the formation of $2 \mathrm{D}$ bands below the edge of the SC bulk 3D band, as the cooperative result of charge redistribution and $(s, p)$ - $d$ hybridization. For these bands, the full exchange splitting occurs in the ferromagnetic state for physically reasonable values of the exchange interaction. Our results also show that the spin-polarized state might be realized as a ferrimagnetic structure resulting from spatially separated FM and SC electron states which are polarized in opposite directions.

The model. - To describe the occurrence of a half-metallic ferromagnetic state near a single FM (sub)monolayer (henceforth, generically layer), we introduce a model which accounts for the relevant degrees of freedom only. In particular, we neglect orbital degrees of freedom, which are responsible for the higher value of the FM spin, resulting from Hund rule within the $d$-orbitals of $t_{g}$ and $e_{g}$ symmetry, but do not play an important role in the formation of a ferromagnetic state. The position vector within our system is $\mathbf{R}=(\mathbf{r}, z)$, with $\mathbf{r}$ parallel and $z$ perpendicular to the layer, which is located at $z=0$. The corresponding wave vector is $\mathbf{K}=(\mathbf{k}, \kappa)$.

For the sake of definiteness, we consider the situation in which the $\mathrm{SC}$ valence band is deep, and can be safely ignored, and the conduction band, arising from a single orbital, taken for simplicity as an $s$-orbital, can be reasonably described, within an effective mass approximation, by the Hamiltonian

$$
\mathcal{H}_{s}=\sum_{\mathbf{k}, \kappa} \sum_{\sigma} \varepsilon_{\mathbf{k}, \kappa} s_{\mathbf{k}, \kappa ; \sigma}^{\dagger} s_{\mathbf{k}, \kappa ; \sigma},
$$

where $s_{\mathbf{k}, \kappa ; \sigma}^{(\dagger)}$ annihilates (creates) an electron with a wave vector $\mathbf{K}=(\mathbf{k}, \kappa)$ and spin projection $\sigma=\uparrow, \downarrow$, and the conduction band is given by

$$
\varepsilon_{\mathbf{k}, \kappa}=\frac{\hbar^{2}}{2 m^{*}}\left(\mathbf{k}^{2}+\kappa^{2}\right) \equiv \varepsilon_{\mathbf{k}}+\varepsilon_{\kappa},
$$

$m^{*}$ being the effective mass. The Hamiltonian of an ideally isolated layer is

$$
\mathcal{H}_{d}=\sum_{\mathbf{k}} \sum_{\sigma} \epsilon_{d} d_{\mathbf{k} ; \sigma}^{\dagger} d_{\mathbf{k} ; \sigma}+I \sum_{i} n_{d, \uparrow}\left(\mathbf{r}_{i}\right) n_{d, \downarrow}\left(\mathbf{r}_{i}\right),
$$

where $d_{\mathbf{k} ; \sigma}^{(\dagger)}$ annihilates (creates) an electron with wavevector $\mathbf{k}$ and $\operatorname{spin} \sigma$ on a $d$-orbital. For simplicity, we assume a single orbital and neglect the small overlap of $d$-orbitals at different sites, taking a dispersionless level $\epsilon_{d}$, the mobility of $d$ electrons being mainly due to the hybridization with the SC s-orbitals. Our model qualitatively corresponds to the real situation in digital alloys such as GaAs/Mn. Indeed, according to the Hund rule for the $\mathrm{Mn}$ ions in a tetrahedral environment, two competing states $\mathrm{Mn}^{3+}\left(d^{4}\right)$ and $\mathrm{Mn}^{2+}\left(d^{5}\right)$, are involved in the hybridization with the $(s p)$-states of the semiconducting host, leading to $e_{2}$ and $t_{2}$ orbitals in the $d^{5}$ configuration, and to $e_{2}$ and $t_{3}$ orbitals in the $d^{4}$ configuration, respectively. The nonbonding $e$-states may be neglected, and the occupation number of a single $d$-orbital in our model corresponds to that of the $t_{2}$ states.

The Coulomb interaction between two electrons in the same $d$-orbital, which is the driving force of magnetism, is described by a Hubbard term of strength $I$. We assume that the electrons are essentially localized at the FM sites $\mathbf{r}_{i}$, and $n_{d, \sigma}\left(\mathbf{r}_{i}\right)$ represents the number of $d$ electrons with spin $\sigma$ on a given site.

When the layer is inserted into the SC matrix, various effects arise. Those which are relevant to the formation of a spin-polarized half-metallic state, as suggested by $a b$ initio calculations, are the SC-FM (here, $s-d$ ) hybridization $V\left(z ; \mathbf{r}-\mathbf{r}^{\prime}\right)$ and the modulation of the local chemical potential $U(z)$, ruling the charge redistribution within the SC matrix around the layer. Both effects preserve the translational invariance along the layer, while breaking the translational invariance along the $z$-axis. The simplest approximation consists in describing these effects as contact terms at $\mathbf{r}=\mathbf{r}^{\prime}$ and $z=0$, corresponding to the Hamiltonians

$$
\begin{aligned}
\mathcal{H}_{s^{-} d} & =\frac{1}{\sqrt{N_{\perp}}} \sum_{\mathbf{k}, \kappa} \sum_{\sigma}\left(V d_{\mathbf{k} ; \sigma}^{\dagger} s_{\mathbf{k}, \kappa ; \sigma}+\text { h.c. }\right), \\
\mathcal{H}_{l o c} & =\frac{U}{N_{\perp}} \sum_{\mathbf{k}, \kappa, \kappa^{\prime}} \sum_{\sigma} s_{\mathbf{k}, \kappa ; \sigma}^{\dagger} s_{\mathbf{k}, \kappa^{\prime} ; \sigma}
\end{aligned}
$$

where $N_{\perp}$ is the number of $\kappa$ values allowed within the first Brillouin zone by the boundary conditions. The first term describes a set of independent one-dimensional Anderson models, labeled by the dummy index $\mathbf{k}$.

We treat the Hubbard term in the Hartree approximation, and adopt the linearization

$n_{d, \uparrow}\left(\mathbf{r}_{i}\right) n_{d, \downarrow}\left(\mathbf{r}_{i}\right) \rightarrow n_{d, \uparrow} n_{d, \downarrow}\left(\mathbf{r}_{i}\right)+n_{d, \downarrow} n_{d, \uparrow}\left(\mathbf{r}_{i}\right)-n_{d, \uparrow} n_{d, \downarrow}$,

where $n_{d, \sigma}=\left\langle n_{d, \sigma}\left(\mathbf{r}_{i}\right)\right\rangle$ is the average number of electrons with spin $\sigma$ in a $d$-orbital. This yields two spin-dependent Hartree $d$ levels $\epsilon_{d, \sigma}=\epsilon_{d}+I n_{d,-\sigma}$. We consider here the case of a generic filling and discard antiferromagnetic or incommensurate order, which is unlikely for an isolated layer, unless particular nesting conditions occur in the layer band structure.

The quantum-mechanical problem associated with the Hamiltonian $\mathcal{H}_{s}+\mathcal{H}_{d}+\mathcal{H}_{s-d}+\mathcal{H}_{\text {loc }}$, after the Hartree decoupling of the Hubbard term, can be dealt with by means of standard techniques, which yield the Green functions

$$
\begin{aligned}
G_{d d}= & \frac{U(1-D) G_{d d}^{0}}{U(1-D)-|V|^{2} D G_{d d}^{0}} \\
G_{s s}\left(\kappa, \kappa^{\prime}\right)= & G_{s s}^{0}(\kappa) \delta_{\kappa, \kappa^{\prime}}+\frac{U}{N_{\perp}} G_{s s}^{0}(\kappa) G_{s s}^{0}\left(\kappa^{\prime}\right) \\
& \times \frac{U+|V|^{2} G_{d d}^{0}}{U(1-D)-|V|^{2} D G_{d d}^{0}}
\end{aligned}
$$


where an implicit diagonal dependence on the wave vector $\mathbf{k}$, on the complex frequency $Z$, and on the spin index $\sigma$ is understood throughout the letter. Here,

$$
\begin{gathered}
G_{d d}^{0}=\frac{1}{Z-\epsilon_{d}}, \\
G_{s s}^{0}(\kappa)=\frac{1}{Z-\varepsilon_{\mathbf{k}}-\varepsilon_{\kappa}}, \\
D=\frac{U}{N_{\perp}} \sum_{\kappa} G_{s s}^{0}(\kappa)=\frac{i u}{\sqrt{Z-\varepsilon_{\mathbf{k}}}},
\end{gathered}
$$

where the square root must be taken with a positive imaginary part, $u \equiv-\frac{\pi}{2} U W_{z}^{-1 / 2}$, and $\left(m^{*} W_{z}\right)^{-1 / 2}$ is a wave vector cutoff. The equation $D=1$ describes $2 \mathrm{D}$ bound states (for $U<0$, i.e., $u>0$ ) or antibound states (for $U>0$, i.e., $u<0$ ) formed near the edge of the $\mathrm{SC}$ conduction band due to the charge redistribution around the layer. These states are localized in the $z$-direction, but propagating along the layer. In this letter, we treat the case $u>0$, when bound states at energies $\bar{\epsilon}_{\mathbf{k}}=\varepsilon_{\mathbf{k}}-u^{2}$ are split off the bottom of the SC conduction band. We do not need in the following the explicit expressions of the $G_{s d}$ and $G_{d s}$ Green functions.

The $s$ - $d$ hybridization removes the poles of the $s$ Green function at bound states $\bar{\epsilon}_{\mathbf{k}}$ and promotes instead 2D $s$ - $d$ bands, which appear as poles of $G_{d d}$ and $G_{s s}\left(\kappa, \kappa^{\prime}\right)$. Analytical expressions can be found for the momentumintegrated Green functions:

$$
\bar{G}_{d d}(Z)=\frac{1}{N_{\|}} \sum_{\mathbf{k}} G_{d d}=\frac{\Phi(Z)-\Phi(Z-W)}{W},
$$

where $N_{\|}$is the number of $\mathbf{k}$-vectors allowed within the first Brillouin zone by the boundary conditions, $W$ is the width of the $2 \mathrm{D}$ band $\varepsilon_{\mathbf{k}}$,

$$
\begin{aligned}
\Phi(\zeta)= & \zeta G_{d d}-2 v G_{d d}^{2}[i \sqrt{\zeta} \\
& \left.-\left(u-v G_{d d}\right) \log \left(u+i \sqrt{\zeta}-v G_{d d}\right)\right]
\end{aligned}
$$

and $v=-|V|^{2} u / U$ (with $U<0$, i.e., $u>0$ and $v>0$ );

$$
\bar{G}_{s s}(Z)=\frac{1}{N_{\|}} \sum_{\mathbf{k}, \kappa} G_{s s}(\kappa, \kappa)=\frac{\Psi(Z)-\Psi(Z-W)}{W}
$$

with

$$
\Psi(\zeta)=\log \frac{u+i \sqrt{\zeta}-v G_{d d}}{i \sqrt{\zeta}}-i \pi N_{\perp} \sqrt{\frac{\zeta}{W_{z}}},
$$

where the last term describes the 3D SC conduction band. The densities of states are then found as

$$
\mathcal{N}_{\alpha}(\omega)=-\frac{1}{\pi} \operatorname{Im} \bar{G}_{\alpha \alpha}(Z=\omega+i \delta),
$$

with $\alpha=s, d$ and $\delta=0^{+}$. In the forthcoming numerical analysis we take $\delta / W=10^{-3}$, two orders of magnitude smaller than the other dimensionless energy scales.
Self-consistent solution. - For numerical simplicity, we work at fixed chemical potential and zero temperature, indicating the Fermi energy with $\varepsilon_{F}$. When the system is spin-polarized, the number of $\uparrow$ and $\downarrow$ spins is different and the source of this unbalance is self-consistently related to the splitting of the $d$ level, $\epsilon_{d, \sigma}=\epsilon_{d}+I n_{d,-\sigma}$. Let us indicate with $\mathcal{N}_{\alpha}^{\sigma}(\omega)$ the density of states for spin $\sigma$, which depends self-consistently on $n_{d,-\sigma}$. Then, the two coupled self-consistency equations which determine $n_{d, \sigma}$ have the form

$$
n_{d, \sigma}=\int_{-\infty}^{\varepsilon_{F}} \mathrm{~d} \omega \mathcal{N}_{d}^{\sigma}(\omega)
$$

By expressing, say, $n_{d, \downarrow}$ as a function of $n_{d, \uparrow}$ and then substituting into the equation for $n_{d, \uparrow}$, the two equations are decoupled, and a single equation of the form $n_{d, \uparrow}=$ $F\left(n_{d, \uparrow}\right)$ is obtained, which can be efficiently solved, e.g., by iterative bisection. Once the self-consistent value of $n_{d, \uparrow}$ is found, one can proceed with the straightforward evaluation of $n_{d, \downarrow}$ and

$$
n_{s, \sigma}=\int_{-\infty}^{\varepsilon_{F}} \mathrm{~d} \omega \mathcal{N}_{s}^{\sigma}(\omega)
$$

Also the thermodynamic grand-canonical potential can be calculated, as

$$
\Omega=\sum_{\alpha=s, d} \sum_{\sigma=\uparrow, \downarrow} \int_{-\infty}^{\varepsilon_{F}} \mathrm{~d} \omega\left(\omega-\varepsilon_{F}\right) \mathcal{N}_{\alpha}^{\sigma}(\omega)-I n_{d, \downarrow} n_{d, \uparrow},
$$

where the last term avoids double counting of the Hartree energy.

To capture the overall qualitative behavior of the numerical results, we adopted the set of parameters $\epsilon_{d} / W=-0.5, u / W^{1 / 2}=1.0$ and $v / W^{3 / 2}=0.05$, without aiming at an accurate fit of the band structure. We then explored the properties of our model for varying $\varepsilon_{F}$ and $I$. Having fixed the zero energy level at the bottom the 3D SC conduction band $\varepsilon_{\mathbf{k}, \kappa} \geqslant 0$, we study only the case $\varepsilon_{F} \leqslant 0$, as there are no free particles in the system to fill the $3 \mathrm{D}$ SC conduction band, so that the chemical potential is, at most, pinned at $\varepsilon_{F}=0$.

To characterize the various phases of our model, we define the partial magnetizations $m_{d}=n_{d, \uparrow}-n_{d, \downarrow}, m_{s}=$ $n_{s, \uparrow}-n_{s, \downarrow}$, and the total magnetization $m_{\text {tot }}=m_{d}+m_{s}$. The resulting phase diagram in the $I / W$ vs. $\left|\varepsilon_{F}\right| / W$ plane is shown in fig. 1. A spin-polarized solution is always found when $I / W$ is sufficiently large. For $\left|\varepsilon_{F}\right| / W \lesssim 0.55$, $s$ spins are antiparallel to $d$ spins (i.e., $\left.m_{d} m_{s}<0\right)$. Since $d$ and $s$ electrons are spatially separated, the system is ferrimagnetic. For larger $\left|\varepsilon_{F}\right|$ the number of majority $s$ spins exceeds the number of minority $s$ spins and the system becomes ferromagnetic (i.e., $m_{d} m_{s}>0$ ). A ferromagnetic ground state is also found when $\left|\varepsilon_{F}\right| / W \lesssim$ 0.25 , in a small region close to the transition to the paramagnetic state. Sharp discontinuities at the 2D band edges produce kinks in the magnetization, and curvature 


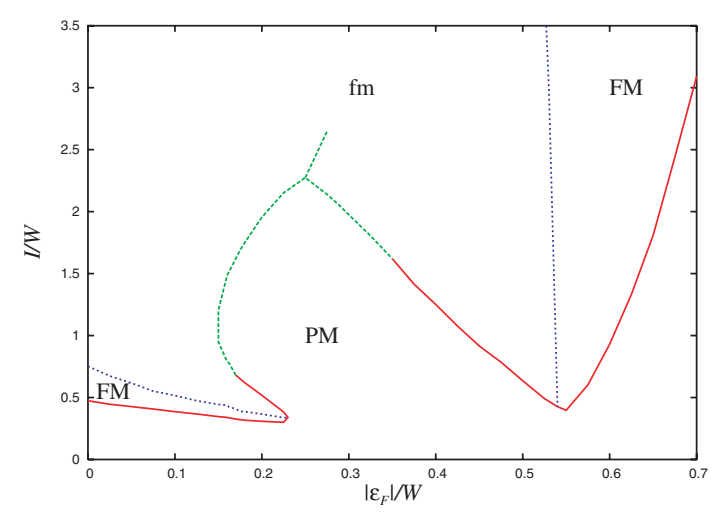

Fig. 1: (Color online) Phase diagram of the model for $\epsilon_{d} / W=$ $-0.5, u / W^{1 / 2}=1$ and $v / W^{3 / 2}=0.05$. The labels PM, FM and $\mathrm{fm}$ indicate the paramagetic, ferromagnetic, and ferrimagnetic phase, respectively. The FM-fm transition and the PM-FM transitions are of second order. The PM-fm transition is of second order for small and large $\left|\varepsilon_{F} / W\right|$, and of first order in an itermediate window. The change of character of the transition line is marked by a different shade.

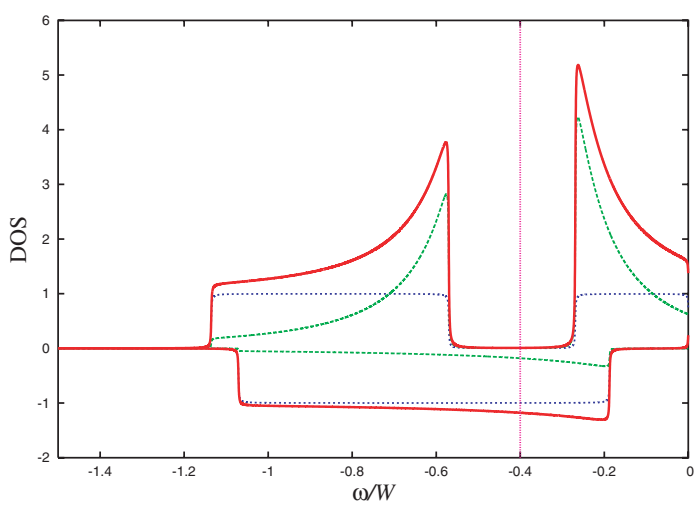

Fig. 2: (Color online) Thick line: Density of states in units of $W^{-1}$ (DOS) as a function of $\omega / W$ for the same parameters as in fig. 1 and $I / W=2, \varepsilon_{F} / W=-0.4$ (marked by a vertical line). The minority-spin DOS is plotted with a negative sign. Thin lines correspond to the $d$ and $s$ contributions, the latter being essentially flat within each band. The system is halfmetallic. The upper minority-spin band is located at $\omega>0$, and is therefore embedded into the 3D SC band.

effects result in a first-order transition and re-entrant phenomena in a window $0.15 \lesssim\left|\varepsilon_{F}\right| / W \lesssim 0.35$.

The spectrum in fig. 2 is taken for $\left|\varepsilon_{F}\right| / W=0.4$ and $I / W=2.0$, and is typical of this region of parameters, where we find a ferrimagnetic half-metallic behavior, with the Fermi level crossing only the lower minority spin 2D band, mainly with $s$ character. The majority spin band is gapped, with the lower band full and the upper band empty, and is therefore insulating. Of course, due to the strong hybridization, a large charge transfer from the $d$ state to the $s$ state occurs in the lower majority-spin band.

This scenario reproduces with fair qualitative agreement, e.g., the behavior of the $\mathrm{Mn} / \mathrm{Si}$ system studied in

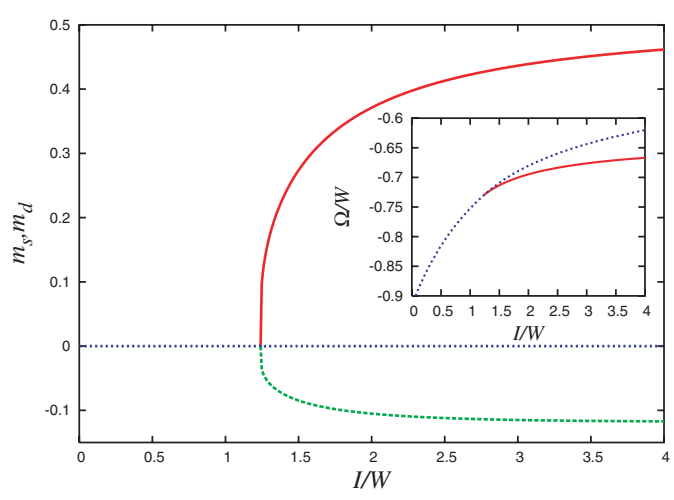

Fig. 3: (Color online) Main panel: $m_{d}$ (upper line) and $m_{s}$ (lower line) for the same parameters as in fig. 1 and $\varepsilon_{F} / W=$ -0.4 . The system is ferrimagnetic ( $\mathrm{fm}$ ) and half-metallic for $I / W>1.2$ and paramagnetic (PM) and insulating for $I / W \leqslant$ 1.2. Inset: Grand-canonical thermodynamic potential for the fm (upper line) and PM (lower line) state. The two lines merge at the second-order transition point.

ref. [6]. Upon reducing $I / W$, the system eventually undergoes a transition to the paramagnetic phase. In fig. 3, we show the behavior of the partial magnetizations $m_{d}$ and $m_{s}$ for $\left|\varepsilon_{F}\right| / W=0.4$, as functions of $I / W$ (in the inset, the behavior of the grand-canonical thermodynamic potential is also shown). The partial magnetizations vanish at the second-order phase transition point, $I / W=1.2$, at which the half-metal becomes an insulator, since the finite density of states at the band edge makes the paramagnetic metal unstable.

The system is half-metallic also for $\left|\varepsilon_{F}\right| / W \lesssim 0,25$, with the Fermi level crossing the upper majority spin band, whereas the lower minority spin band is full and insulating.

We point out that the driving force of magnetism is the Coulomb repulsion $I$ on the $d$-orbitals. To understand the magnetic phases in the phase diagram of fig. 1, we can consider the case when $I$ is sufficiently large, so that the $d$ level of majority spins is full and the $d$ level of minority spin is empty. Then, the $s-d$ hybridization shifts only the majority-spin bound-state $s$ band from its paramagnetic position, and two bands are formed for the majority spins, with strong $s$ - $d$ mixing, whereas the minority-spin $s$ band stays almost unchanged and keeps a pure $s$ character. The sign of the shift of the majority-spin $s$ band depends on the relative positions of the majority-spin $d$ level (e.g., $\epsilon_{d, \uparrow}$ in the case of up majority spins) and the center of the bound-state $\mathrm{s}$ band in the paramagnetic phase $(\bar{\epsilon})$. If $\epsilon_{d, \uparrow}>\bar{\epsilon}$, as it is the case in fig. 1, with lowering the Fermi level, the situation is reached when the number of majority $s$ spins exceeds the number of minority $s$ spins, and the $s-d$ spin alignment changes from antiferromagnetic (leading to global ferrimagnetism) to ferromagnetic. In the opposite situation, when $\epsilon_{d, \uparrow}<\bar{\epsilon}$, a regime where the majority-spin $s$ band is shifted to higher energy is easily reached, so that, with lowering the Fermi level, the $s$ - $d$ spin alignment changes from ferromagnetic to antiferromagnetic. 
Concluding remarks. - Our Anderson-like model captures the main ingredients of the physics of a FM layer in a SC host, and is therefore able to account for the occurrence of a spin-polarized half-metallic behavior in these systems. This is the cooperative result of charge redistribution and confinement around the FM layer and FM-SC hybridization, giving rise to $2 \mathrm{D}$ bands which undergo a full exchange splitting for physically reasonable values of the exchange coupling.

Of course, our model misses some aspects of the numerical calculations, such as the orbital dengeneracy, the crystal-field splitting, and details of the lattice band structure beyond the effective mass approximation. These aspects can be accounted for in a straightforward way, by proper generalizations of the starting model, equipping the various lattice bands with orbital labels, and considering the various hybridizations of $(s, p)$ and $d$ bands, allowed by symmetry. However, the subsequent analysis of the model would require much heavier numerical effort, making the results less transparent.

Further lines of investigation concern the role of quantum and thermal fluctuations and the mechanism of exchange coupling among different FM layers, when these are organized in a periodic DMH structure.

As far as the first aspect is concerned, we have preliminarly checked that quantum fluctuations of the magnetization, both on the metallic and on the insulating side of the ferromagnetic-paramagnetic transition are not strong enough to wash out the mean-field scenario even for an isolated layer. Spatial fluctuations of the magnetization are characterized by a $|\mathbf{q}|^{2}$ term which arises from the expansion of the $d-d$ susceptibility at small wave vectors of the 2D (intra-layer) fluctuations, within the randomphase approximation. The dynamics of the fluctuations is ruled by a $(i \omega /|\mathbf{q}|)$-term on the metallic side, due to Landau damping, and by a $\omega^{2}$-term on the insulating side. In both cases, the fluctuation correction to the meanfield critical value of the Hubbard interaction $I$ is finite, although analytical calculations cannot be pushed too far, and a numerical analysis is required to provide quantitative results. Of course, fluctuations are even weaker in the case of a 3D DMH.

To evaluate the exchange interaction between two FM layers, in ref. [7] we used a phenomenological model which assumed ferromagnetic order within each layer, and showed that there are two main mechanism, which lead to the competition of ferromagnetic and antiferromagnetic coupling. The first interaction, mediated by mobile electrons in the 2D bound-state bands, is ferromagnetic when the FM layers are close enough, but changes sign with increasing inter-layer distance, becoming antiferromagetic. A further change of sign, back to ferromagnetic, is also possible at even larger distances, depending on the values of the parameters. The second interaction, mediated by inter-band virtual transitions between 3D bands, may oscillate with the distance, and is modulated by an exponentially decreasing prefactor. The case of a periodic array of FM layers could be studied within a suitable generalization of the model presented in this letter, which would allow to treat intra- and inter-layer exchange on an equal footing.

The work was partially supported by the University of the Basque Country (9/UPV 00206.215-13639/2001), the Basque Unibertsitate eta Ikerketa Saila, the Spanish Ministerio de Ciencia y Tecnología (FIS 2004-06490C03-01), and by RFBR (Grant No. 07-02-00114-a).

\section{REFERENCES}

[1] Nazmul A. M., Sugahara S. and Tanaka M., Phys. Rev. B, 67 (2003) 241308(R); NAzmul A. M., Kobayashi S., Sugahara S. and Tanaka M., Physica E, 21 (2004) 937; Aronzon B. A., Lagutin A. S., Ryl'kov V. V., Tugushev V. V., Men'shov V. N., Lashkul A. V., Laiho R., Vikhrova O. V., Danilov Yu. A. and Zvonkov B. N., JETP Lett., 87 (2008) 164.

[2] Luo H., McCombe B. D., Na M. H., Mooney K., Lehmann F., Chen X., Cheon M., Wang S. M., Sasaki Y., Liu X. and Furdyna J. K., Physica E, 12 (2002) 366; Chen X., Na M., Cheon M., Wang S., Luo H., MCCombe B. D., Liu X., SASAKi Y., Wojtowicz T., Furdyna J. K., Potashnik S. J. and Schiffer P., Appl. Phys. Lett., 81 (2002) 511; McCombe B. D., NA M., Chen X., Cheon M., Wang S. M., Luo H., Liu X., Sasaki Y., Wojtowicz T., Furdina J. K., Potashnik S. J. and Schiffer P., Physica E, 16 (2003) 90.

[3] Sanvito S. and Hill N. A., Phys. Rev. Lett., 87 (2001) 267202; Qian Gefei, Chang Yia-Chung and Tucker J. R., Phys. Rev. B, 71 (2005) 012414; QIAN M. C., Fong C. Y. and Pickett W. E., J. Appl. Phys., 99 (2006) 08D517; Zhou X. H., Chen Xiaoshuang, Huang Y., Duan H., Sun L. Z. and Lu W., J. Appl. Phys., 99 (2006) 113903.

[4] Continenza A., Antoniella F. and Picozzi S., Phys. Rev. B, 70 (2004) 035310; WANG H.-Y. and QIAN M. C., J. Appl. Phys., 99 (2006) 08D705.

[5] Qian M. C., Fong C. Y., Liu Kai, Pickett W. E., Pask J. E. and Yang L. H., Phys. Rev. Lett., 96 (2006) 027211.

[6] Wu H., Kratzer P. and Scheffler M., Phys. Rev. Lett., 98 (2007) 117202.

[7] Men'shov V. N., Tugushev V. V., Echenique P. M., Caprara S. and Chulkov E. V., Phys. Rev. B, 78 (2008) 024438. 